\title{
Relação entre o alinhamento pélvico e a incontinência urinária de esforço
}

\author{
Sandra Beatriz Aires dos Santos ${ }^{1}$ Hedioneia Maria Foletto Pivetta² Ana Fátima Viero Badaró ${ }^{3}$
}

\begin{abstract}
RESUMO
O equilíbrio da pelve é essencial para a continência urinária. Neste estudo buscou-se verificar a relação entre a Incontinência Urinária de Esforço (IUE) e o alinhamento pélvico em mulheres adultas. 0 trabalho constituiu na aplicação de um questionário adaptado, específico em Uroginecologia e exames de Raios-X em uma amostra constituída por 25 mulheres, divididas em dois grupos, o Grupo Controle (GC) e 0 Grupo Experimental (GE), com idade entre 40 e 57 anos. Os resultados foram submetidos a testes estatísticos, com nível de significância de $5 \%$. Os resultados sugerem relação entre alterações de mais de um ângulo pélvico, o que sugere prejuízo do alinhamento pélvico e, consequentemente predisposição a incontinência urinária de esforço (IUE). Não houve correlação de alteração no alinhamento pélvico em um ângulo isoladamente.
\end{abstract}

Descritores: Pelve; Incontinência Urinária; Saúde da Mulher; Radiografia.

\section{Relationship between pelvic alignment and the stress urinary incontinence}

\begin{abstract}
The equilibrium of the pelvis is essential for urinary continence. This study aimed to verify the relationship between urinary incontinence (SUI) and pelvic alignment in adult women. The study consisted of applying a tailored, specific questionnaire in Urogynecology, and X-ray examinations in a sample of 25 women divided into two groups: control (CG) and experimental (EG) aged 40 to 57 years. The results were submitted to statistical tests with significance level of $5 \%$. The results suggest a relationship between changes over a pelvic angle, suggesting loss of pelvic alignment and hence a predisposition to stress urinary incontinence (SUI). There was no correlation between change in pelvic alignment at an angle alone.
\end{abstract}

Descriptors: Pelvis; Urinary Incontinence; Women's Health; Radiography

\footnotetext{
${ }^{1}$ Especialista em Reabilitação físico-motora pela Universidade Federal de Santa Maria (UFSM), Santa Maria, RS, Brasil.

2 Doutora em Educação pela Universidade Federal de Santa Maria (UFSM), Santa Maria, RS, Brasil.

${ }^{3}$ Pós doutora em Ética e bioética pela Universidad Complutense de Madrid (UCM), Madrid, Espanha.
} 


\section{Introdução}

Incontinência urinária (IU) é um problema que afeta mulheres de todas as idades ${ }^{1}$. A Incontinência Urinária de Esforço (IUE) é definida como uma perda involuntária de urina pela uretra, anatomicamente normal, resultante de uma disfunção do equilíbrio vésico-esfincteriano, quando a pressão vesical excede a pressão uretral máxima, na ausência de atividade do músculo detrusor, ocorrendo, em regra, por aumentos da pressão intra-abdominal². Essa disfunção acaba por causar problemas sociais e/ou de higiene na pessoa, tornando-se de suma importância seu estudo e a discussão das alternativas de tratamento.

A IUE é considerada multifatorial e apresenta como fatores de risco o parto traumático, com o uso de fórceps e/ ou episiotomias; deficiência estrogênica, condições associadas ao aumento da pressão intra-abdominal; tabagismo; diabetes; neuropatias e histerectomia prévia ${ }^{3}$, sendo que o bom equilíbrio da bacia pélvica depende da condição postural ${ }^{4}$.

A postura estática é mantida pela integração dos músculos que funcionam como estabilizadores do corpo, contraindose apenas para manter a harmonia articular, permitindo que 0 indivíduo permaneça em ortostase ${ }^{5}$. Em posição ereta surgem, portanto, as consequências das retrações de músculos da estática ${ }^{6}$. A manutenção de uma postura correta da região pélvica torna-se um fator de contribuição para a continência nas situações de aumento da pressão intraabdominal? .

Os desequilíbrios pélvicos podem levar a um déficit de força da musculatura perineal e colaborar negativamente para a continência, já que o mecanismo esfincteriano também estará comprometido ${ }^{8}$. A continência urinária depende do equilíbrio entre as forças de expulsão e as de retenção (tonicidade dos esfíncteres lisos e estriados e resistência uretral) e da posição intrapélvica da uretra proximal em relação ao colo vesical, onde os aumentos da pressão intraabdominal devem ser transmitidos diretamente à uretra ${ }^{9}$. As fibras do músculo levantador do ânus, ligadas à fáscia endopélvica formam uma rede de sustentação muito importante para a manutenção da continência, pois durante a contração muscular, por circundar a vagina e a porção distal da uretra, essa rede vai tracionar a uretra em direção ao púbis e comprimi-la contra a parede vaginal, mantendo, assim, a luz uretral ocluída ${ }^{10}$.

Diante do exposto, este estudo buscou verificar a relação entre a Incontinência Urinária de Esforço (IUE) e o alinhamento pélvico em mulheres adultas.

\section{Metodologia}

A amostra foi composta por 25 mulheres, com diagnóstico de IUE, na faixa etária dos 40 aos 57 anos, provenientes do Ambulatório de Ginecologia e do Ambulatório de Fisioterapia de um hospital-escola, da rede pública federal, do sul do Brasil. As participantes foram divididas em dois grupos, um denominado Grupo Controle (GC), com 13 mulheres continentes e o outro, denominado Grupo Experimental (GE), com 12 mulheres com diagnóstico urodinâmico de IUE.

O estudo apresenta abordagem analítica observacional e delineamento transversal ${ }^{11}$, com Grupo Controle. Foi aprovado pelo Comitê de Ética em Pesquisa (CEP) da instituição responsável, sob o protocolo, número 263.224/2013. Previamente, ao início da coleta de dados, as voluntárias assinaram o Termo de Consentimento Livre e Esclarecido (TCLE).

A coleta de dados se deu entre os meses de maio a outubro de 2013, através do preenchimento de questionário adaptado, específico em Uroginecologia ${ }^{12}$,, com o objetivo de delinear o perfil uroginecológico das mulheres avaliadas. Após, realizaram-se exames radiológicos da pelve, nas posições perfil e ântero-posterior, ambos em ortostase.

O alinhamento pélvico, em perfil, foi analisado, de acordo com Rolf ${ }^{13}$, que considera uma pelve equilibrada aquela em que a linha entre o púbis e cóccix (A-A') estará na horizontal formando um ângulo reto $\left(90^{\circ}\right)$, com a linha vertical (B-B') entre o púbis e a espinha ilíaca ântero-superior e um ângulo de $60^{\circ}$, com a linha traçada entre o cóccix e a $5^{a}$ vértebra lombar ( $\left.C-C^{\prime}\right)$. Desse modo, foram mensurados e analisados os ângulos $X\left(A-B^{\prime}\right)$ e $Y\left(A^{\prime}-C\right)$, conforme observados na figura 1. Ainda na vista anterior, a simetria da pelve foi verificada pela horizontalidade das EIAS (linha $\left.E-E^{\prime}\right)$ e por uma linha vertical que passa entre a $5^{a}$ vértebra lombar e a sínfise púbica (F-F') formando um ângulo reto (ângulo $\left.Z=90^{\circ}\right) 13$, como apresentado na figura 2 . 


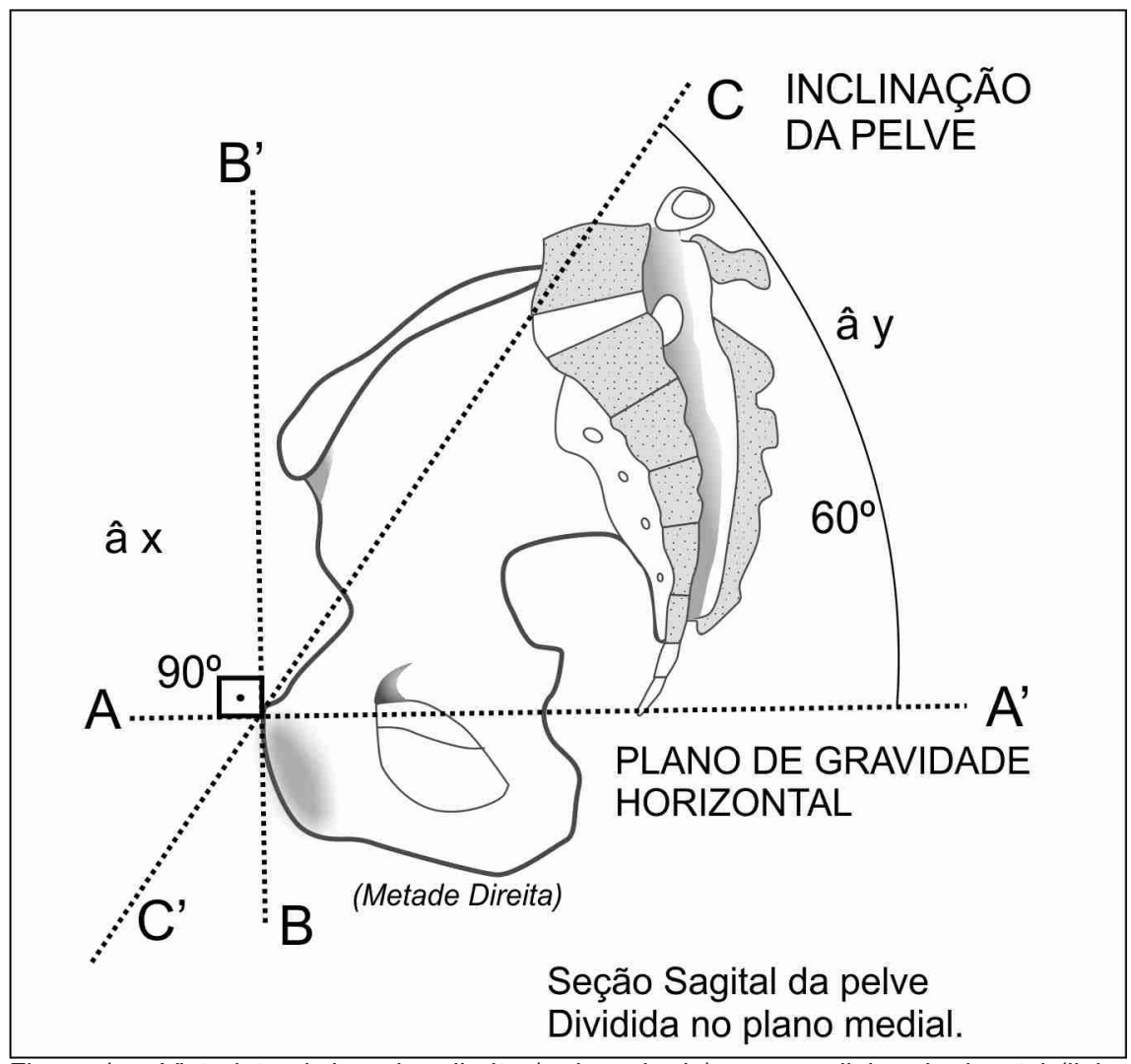

Figura 1 - Vista lateral da pelve direita (redesenhada), com as linhas horizontal (linha A-A'), entre o cóccix e o púbis; vertical (linha B-B'), entre o púbis e espinha ilíaca ânterosuperior (EIAS); e a linha traçada entre o cóccix e a $5^{a}$. vértebra lombar (linha C-C') e os ângulos X e Y. Fonte: ROLF, 1990, p.86.

Os exames de RX e as análises dos ângulos da pelve foram realizados por um técnico em radiologia. Foi utilizado 0 Software Animati Workstation, uma ferramenta de visualização avançada, desenvolvida pela empresa Animati Sistema de Arquivamento e Comunicação de Imagens Diagnósticas (PACS), para análise de RX digital, com fins diagnósticos (Figura 3).

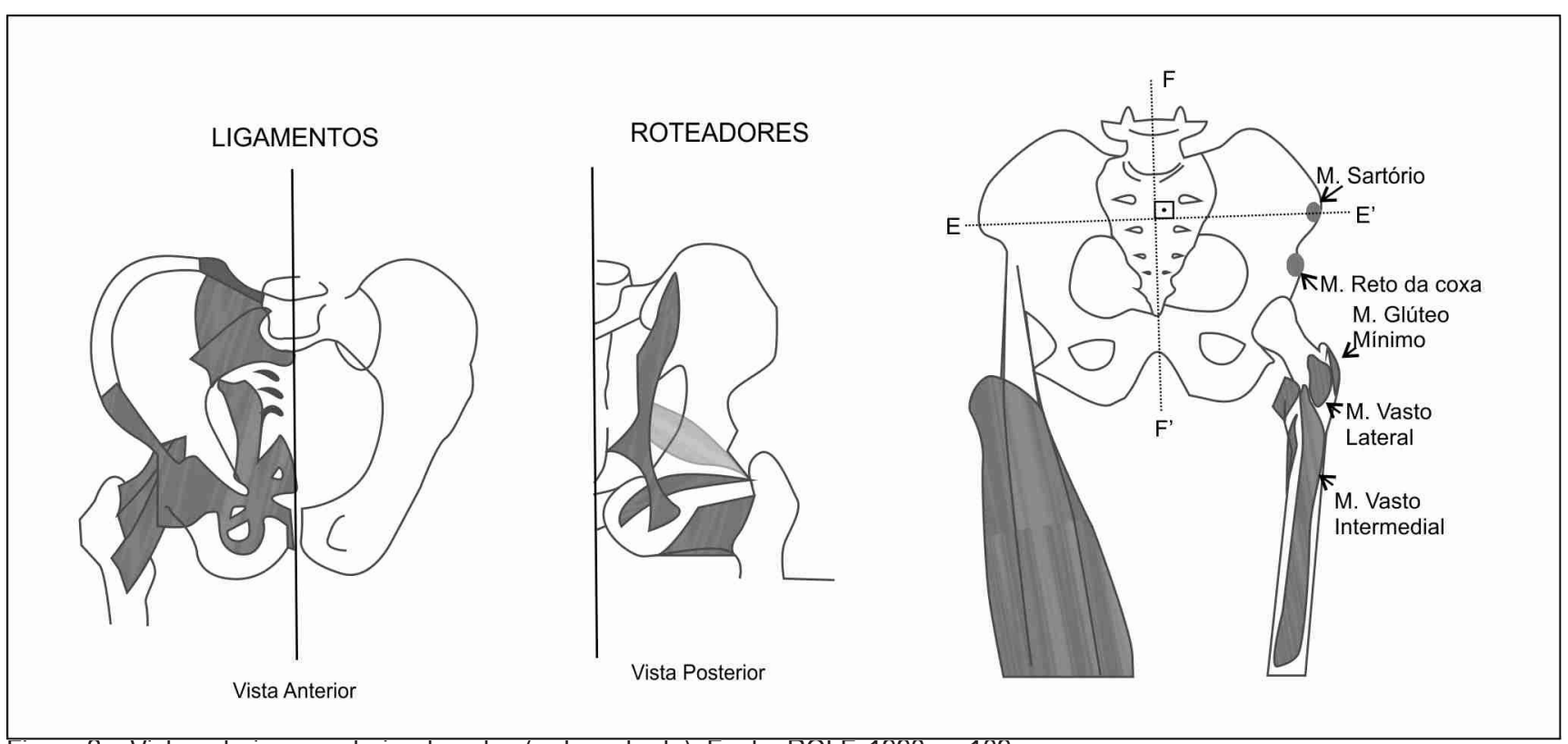

Figura 2 - Vista anterior e posterior da pelve (redesenhada). Fonte: ROLF, 1990, p. 109. 

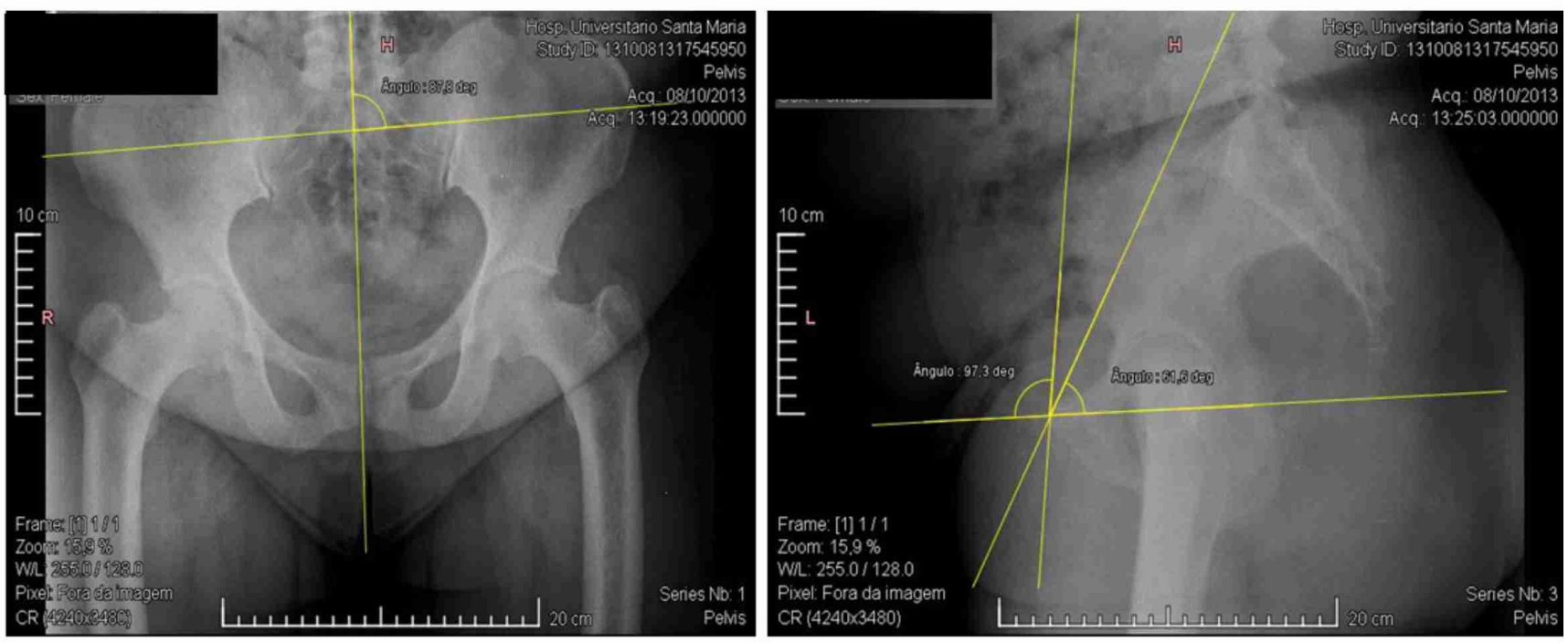

Figura 3 - Pelve em vista ântero-posterior e perfil gerada pelo software Animati Workstation

Foram excluídas do estudo as mulheres submetidas a cirurgias prévias, afecções musculoesqueléticas, reumatológicas e escoliose estrutural, referidas e/ou detectadas, após o encaminhamento médico, e aquelas com imagens radiológicas com presença de fixação óssea metálica nos membros inferiores, pelve e/ou coluna vertebral. Três pacientes foram agendadas e não compareceram ao exame e houve uma desistência em decorrência da morosidade na realização do exame. Posteriormente, foram excluídas do GC cinco imagens radiográficas digitalizadas, por apresentarem medidas imprecisas, devido à baixa qualidade visual.

A análise estatística dos dados foi realizada por meio dos testes: Shapiro Wilk, para testar a normalidade dos dados, Teste "t", para amostras independentes, Qui-quadrado e correlação de Pearson. Para o estudo das frequências das variáveis "tipo de parto" e "escolaridade" foi utilizada a estatística descritiva de frequência. Também foi utilizado 0 aplicativo SPSS for Windows ${ }^{\circledR}$, versão 14.0 e adotado um nível de significância de 5\%.

\section{Resultados}

A apresentação dos resultados foi organizada a partir da caracterização da amostra, em que são consideradas as variáveis idade, IMC, tipo de parto e escolaridade. Na sequência, apresentam-se as angulações obtidas na avaliação do alinhamento pélvico das mulheres com e sem IUE.

O GC (n=13) apresentou média de idade de 50,3 anos e IMC de 29,8 $\mathrm{Kg} / \mathrm{cm} 2 ; 76,9 \%$ são da cor branca e 30,7\% são solteiras. Quanto à profissão, encontrou-se: 15,4\%, empregada doméstica; $23,1 \%$, serviços gerais; $15,4 \%$, do lar; $15,4 \%$, trabalhadoras do comércio (vendedoras); $15,4 \%$, cabeleireira; 7,7\%, costureira, e 7,7\%, técnica em enfermagem. Já, no GE (n=12), a média de idade foi de 50,2 anos e IMC de $27,7 \mathrm{Kg} / \mathrm{cm} 2 ; 83,3 \%$ são da cor branca e todas são casadas. A profissão verificada neste grupo foi $75 \%$, do lar; $16,6 \%$, serviços gerais e $8,3 \%$, agricultoras.

As mulheres do GC tiveram 46 gestações, 37 partos e 12 abortos, e as do GE tiveram 31 gestações, 28 partos e sete abortos. Quanto ao tipo de parto, o GC apresentou 38,4\% de ambos os tipos de parto, seguido de 30,8\% de partos vaginais e $30,8 \%$ de partos cesáreos, sendo que cinco delas tiveram mais de dois partos. No GE, $50 \%$ tiveram parto vaginal; $25 \%$ foram submetidas a parto cesáreo, e $25 \%$ a ambos, sendo que três delas tiveram mais de dois partos. A Tabela 1 apresenta os tipos de parto, as frequências e a comparação intergrupos.

\begin{tabular}{c|c|c}
\multicolumn{2}{c}{ Tabela 1 - Comparação entre tipo de parto, por mulher, do GC e GE } \\
\hline Tipos de parto & \multicolumn{2}{|c}{ Frequência } \\
\hline & Grupo GC n $(\%)$ & Grupo GE n $(\%)$ \\
\hline Vaginais & $4(30,8)$ & $6(50,0)$ \\
\hline Cesarianas & $4(30,8)$ & $3(25,0)$ \\
\hline Ambos & $5(38,4)$ & $3(25,0)$ \\
\hline
\end{tabular}

n=número de sujeitos 
Quanto à escolaridade, no GC, predominou mulheres com ensino fundamental incompleto (46,1\%), seguido do ensino médio completo (23,1\%). Entretanto, no GE imperou o ensino médio incompleto (75\%), o que pode ser observado na Tabela 2.

Tabela 2 - Escolaridade das participantes do GC e GE

\begin{tabular}{c|c|c}
\hline Nível & \multicolumn{2}{|c}{ Frequência } \\
\hline Ensino fundamental incompleto & GC n (\%) & GE n $(\%)$ \\
\hline Ensino fundamental completo & $7(46,1)$ & $1(8,3)$ \\
\hline Ensino Médio ou antigo 2a grau incompleto & $1(7,7)$ & $2(16,7)$ \\
\hline Ensino Médio ou antigo 2a grau completo & $4(23,1)$ & $9(75,0)$ \\
\hline Superior incompleto & $1(7,7)$ & $0(0)$ \\
\hline Pós-graduação & $1(7,7)$ & $0(0)$ \\
\hline n=número de sujeitos. & \multicolumn{2}{|c}{}
\end{tabular}

O alinhamento pélvico em perfil foi verificado pelas medidas dos ângulos $X$ e Y e, na vista anterior, pelo ângulo Z. A análise descritiva das medidas é apresentada na Tabela 3, de acordo com as angulações que caracterizam o alinhamento pélvico normal para os ângulos X, Y e Z. A Tabela 4 apresenta as médias dos ângulos X, Y e Z do GC e GE, o Teste te o nível de significância

Tabela 3 - Análise descritiva dos ângulos do alinhamento pélvico em perfil (â X e â Y) e na vista anterior (â Z)

\begin{tabular}{c|c|c|c|c|c}
\hline Grupos e ângulos & $\mathrm{n}$ & Mínimo & Máximo & Média & DP \\
\hline GC â X $\left(90^{\circ}\right)$ & 13 & 90,50 & 109,80 & 97,06 & 5,68 \\
\hline GC â Y $\left(60^{\circ}\right)$ & 13 & 50,70 & 68,80 & 59,92 & 4,64 \\
\hline GC â Z $\left(90^{\circ}\right)$ & 13 & 87,60 & 91,60 & 89,56 & 1,18 \\
\hline GE â X $\left(90^{\circ}\right)$ & 12 & 86,80 & 99,90 & 94,35 & 4,22 \\
\hline GE â Y $\left(60^{\circ}\right)$ & 12 & 52,40 & 96,20 & 65,60 & 10,77 \\
\hline GE â Z $\left(90^{\circ}\right)$ & 12 & 66,30 & 91,80 & 87,74 & 6,86 \\
\hline
\end{tabular}

Tabela 4 - Médias dos ângulos X, Y e Z para os GC e GE

\begin{tabular}{c|c|c|c|c}
\hline Ângulos & $\begin{array}{c}\text { GC } \\
\text { (média e DP) }\end{array}$ & $\begin{array}{c}\text { GE } \\
\text { (média e DP) }\end{array}$ & T & $P$ \\
\hline$X$ & $97,06 \pm 5,60$ & $94,35 \pm 4,22$ & 1,353 & 0,425 \\
\hline$Y$ & $59,92 \pm 4,64$ & $65,60 \pm 10,77$ & $-1,738$ & 0,244 \\
\hline$Z$ & $89,56 \pm 1,18$ & $87,74 \pm 6,86$ & 0,943 & 0,102 \\
\hline
\end{tabular}

$O$ teste de Pearson para correlacionar o alinhamento pélvico com a IUE mostrou correlação negativa forte $(r=-0,919$, $p=0,000)$ no $G E$, nos ângulos $Z$ e $Y$ e correlação negativa moderada $(r=-0,605, p=0,037)$ no GE, ângulos $X$ e $Y$.

\section{Discussão}

A amostra caracterizou-se por mulheres, com média de idade de 50 anos e IMC elevado $\left(G C=29,1 \mathrm{Kg} / \mathrm{cm}^{2}\right.$ e GE = $\left.27,7 \mathrm{Kg} / \mathrm{cm}^{2}\right)$, o que indica, segundo a Organização Mundial da Saúde (OMS, 2004), que tanto as mulheres do GC como aquelas do GE estão com sobrepeso.

Em pesquisa que avaliou a influência da menopausa no IMC em 1.506 mulheres, entre 20 e 59 anos, no Rio de Janeiro, observou-se que, aproximadamente $40 \%$ delas, apresentaram algum grau de sobrepeso ${ }^{14}$. Em outro estudo realizado na população urbana do município de ljuí-RS, verificou-se que a incontinência urinária é uma situação que se associa ao grau de obesidade. $O$ estudo evidenciou que quanto maior o grau de obesidade, maior a frequência de IUE e que, com 0 aumento do IMC, a frequência de IU também aumenta. Os autores verificaram, ainda, que o grau de obesidade aumenta a incidência de hipertensão arterial sistêmica, diabetes mellitus, doença cardiovascular e incontinência urinária ${ }^{15}$. 
Estudos indicam que a baixa escolaridade influencia na demora em procurar o tratamento para a doença, uma vez que pode haver o entendimento de que a IU é uma consequência natural do processo de envelhecimento ${ }^{16}$. Estimativas demonstram que apenas $50 \%$ das pessoas com IU procuram o serviço de saúde por este motivo, o que leva à conclusão que a baixa escolaridade de idosos é um dos fatores de risco para IU ${ }^{17}$.

Nesta pesquisa, os dados referentes à escolaridade verificaram que 46,6\% das mulheres do GC apresentam ensino fundamental incompleto, ou seja, menos de cinco anos de estudo, e $75 \%$ do GE tinham ensino médio incompleto, o que demonstra que as mulheres com IUE, que apresentaram maior nível de escolaridade, foram as que procuraram o serviço de saúde.

Quanto ao número de gestações e partos pode-se inferir que o GC apresentou maior número de ambos. Contudo, a variável, tipo de parto, mostra que no GE, $50 \%$ deles foram vaginais, enquanto no GC foi de $30,8 \%$, sugerindo que este seja um fator de risco iminente para a IU neste grupo.

A literatura refere que tanto a gestação quanto o parto são considerados fatores de risco para a IU, pois proporcionam mudanças anatômicas e funcionais do sistema de sustentação pélvica ${ }^{18}$. As elevações bruscas da pressão intraabdominal constituem fatores de risco de IUE, o que ocorre no período gestacional e é intensificado no momento do parto vaginal ${ }^{18}$. Estudos sugerem que a prevalência de IU é maior entre mulheres com antecedente de algum tipo de parto, ainda que este tenha sido cesáreo, o que sugere ser o trabalho de parto o principal fator associado à ocorrência dessa queixa ${ }^{19,20}$.

A pelve equilibrada implica no nivelamento da ponta do cóccix ao púbis, visto no plano sagital, e no nivelamento das EIAS no plano frontal|13,21,22, ou seja, em simetria das hemipelves, o que contribui para a sustentação dos órgãos pélvicos. Neste estudo, tanto o GC quanto o GE apresentaram maior inclinação pélvica, demonstrado pelo aumento do ângulo $X$, enquanto que o ângulo $Y$ apresentou-se alterado somente no $\operatorname{GE}\left(65,6^{\circ}\right)$. Isto pode indicar que a inclinação da pelve contribui para a IU neste grupo. Ainda, no GE, observou-se diminuição do ângulo $Z\left(87,74^{\circ}\right)$, o que significa a rotação da pelve, e que ratifica o desalinhamento dessa estrutura.

$\mathrm{Na}$ correlação das variáveis que procurou identificar as alterações angulares, os resultados não apresentaram significância estatística para amostras independentes em ambos os grupos, ou seja, a alteração de um ângulo isoladamente não está associada ao fator IUE. Entretanto, quando há associação de alterações de angulações (Z e Y; X e Y), os testes demonstraram forte correlação para a IUE.

Em estudo que buscou a associação do posicionamento pélvico e da lordose lombar com a IUE, não foi encontrada diferença significativa na simetria da pelve ou na lordose lombar nas mulheres que apresentavam a disfunção ${ }^{23}$. No mesmo estudo, a análise da báscula de pelve, por fotogrametria, indicou tendência à anteversão pélvica entre as mulheres do grupo controle sem IUE. No conjunto da amostra, apenas uma fraca correlação foi encontrada entre a medida da lordose lombar, por fotogrametria, e o ângulo sacral medido por radiografia ${ }^{23}$. Não foram encontrados outros estudos que investigaram o alinhamento pélvico com $\mathrm{RX}$.

\section{Conclusão}

Os resultados sugerem relação entre alterações angulares associadas, entre si, o que se refere ao prejuízo do alinhamento pélvico e a incontinência urinária de esforço (IUE). Não houve correlação de alteração no alinhamento pélvico em um ângulo isoladamente.

Sugere-se a investigação do alinhamento pélvico de mulheres com IU, averiguando-se, também, a angulação sacral e lombar, além de investigar possíveis alterações da postura corporal e sua relação com o alinhamento da pelve. Também é interessante relacionar fatores de risco na gestação e tipo de parto, com a presença de IUE.

\section{Agradecimentos:}

À Mateus Silva Trindade, técnico em radiologia que realizou os exames de imagem e a mensuração dos ângulos pélvicos. 


\section{Referências Bibliográficas}

1. Faria K, Pedrosa LAK. Avaliação da qualidade de vida e função sexual de mulheres com e sem incontinência urinária. Rev. Eletr. Enf. [Internet]. 2012 Abr-Jun; 14(2):366-73. Disponível em: http://www.fen.ufg.br/revista/v14/n2/ v14n2a17.htm.

2. Oliveira E, Zuliani LMM, Ischicava J, Silva SV, Albuquerque SSR, Souza, AMB, Barbosa PC. Avaliação dos fatores relacionados à ocorrência da incontinência urinária feminina. Rev Assoc Med Bras. 2010; 56(6):688-690.

3. Guarisi T, Pinto-Neto, AM, Osis MJ, Pedro AO, Costa-Paiva LHS, Fagundes A. Procura de serviço médico por mulheres com incontinência urinária. Rev Bras Ginecol Obstet. 2001; 23(7):439-43.

4. Etiene MA, Watman MC. Disfunções sexuais femininas. São Paulo: LMP, 2006.

5. Santos, A. Diagnostico Clinico Postural: um guia prático. 6ª Edição. São Paulo: Summus; 2011.

6 . Souchard PE. Le rôle de la rééducation posturale globale (RPG) dans les pathologies respiratoires. Créateur de la RPG, Université de Thérapie Manuelle. Rev Mal Respir. 2005; 22.:524-5.

7. Bienfait M. Fisiologia da Terapia Manual. São Paulo: Summus; 2000.

8. Perry JD, Hullet TL. Urinary incontinence and pelvic muscle rehabilitation index. Northeastern Gerontological Society. New Jersey: New Brunswick; 1998.

9. Rodrigues NC, Scherma D, Mesquita RA, Oliveira J. Exercícios perineais, eletroestimulação e correção postural na incontinência urinária - estudo de casos. Rev Fisioter Mov. 2005 Jul-Set; 18(3)23-29.

10. Matheus LM, Mazzari CF, Mesquita RA, Oliveira J. Influência dos exercícios perineais e dos cones vaginais, associados à correção postural, no tratamento da incontinência urinária feminina. Braz. J. Phys. Ther. 2006 Out-Dez; 10(4)387-392.

11. Marques AP, Peccin MS. Pesquisa em Fisioterapia: a prática baseada em evidências e modelos de estudos. Fisioter. Pesqui. 2005;11(1):43-8.

12. Souza GA, Carvalho RS. Avaliação da capacidade de contração muscular do assoalho pélvico feminino em diversas posições. Trabalho de Conclusão de Curso (Bacharel em Fisioterapia) - Universidade da Amazônia, Belém do Pará. 2007.

13. Rolf IPR. Rolfing: a integração das estruturas humanas. São Paulo: Martins Fontes;1990.

14. Sichieri R, Lins APM. Influência da menopausa no índice de massa corporal. Instituto de Medicina Social, Rio de Janeiro, RJ. Arq Bras Endocrinol Metab. 2001; 45(3). p. 265-70.

15. Rasia J, Berlezi EM, Bigolin SE, Schneider RH. A relação do sobrepeso e obesidade com desconfortos musculoesqueléticos de mulheres pós-menopausa. Revista Brasileira de Ciências do Envelhecimento Humano (RBCEH). 2007; 4(1):28-38.

16. Brasil. Ministério da Saúde. Secretaria de Atenção à Saúde. Departamento de Atenção Básica. Atenção à saúde da pessoa idosa e envelhecimento. Cadernos de Atenção Básica, Brasília, n. 19, Série A. Normas e Manuais Técnicos - 2010.

17. Bolina AF, Dias FA, Santos NMF, Tavares DMS. Incontinência urinária autorreferida em idosos e seus fatores associados. Rev Rene. 2013; 14(2):354-63.

18. Reis AO, Câmara CNS, Santos SG, Dias TS. Estudo comparativo da capacidade de contração do assoalho pélvico em atletas de voleibol e basquetebol. Rev Bras Med Esporte. 2011 Mar-Apr; 17(2) p. 97-101.

19. Hunskaar S, Arnold EP, Burgio K, Diokno AC, Herzog, AR, Mallett VT. Epidemiology and natural history of urinary incontinence. Int Urogynecol J Pelvic Floor Dysfunct. 2000;11:301-19.

20. Borges JBR, Guarisi T, Camargo ACM, Gollop TR, Machado RB, Borges PCG. Incontinência urinária após parto vaginal ou cesáreo. Einstein (Säo Paulo). 2010; 8(2), Abr.-Jun. graf, tab.: 192-96.

21. Bienfait M. Fisiologia da Terapia Manual. São Paulo: Summus; 1987.

22. Kapandji Al. Fisiologia Articular: esquemas comentados de mecânica humana. 6a ed. São Paulo: Guanabara Koogan, 2007.

23. Araújo THP, Francisco TLTP, Leite RF, lunes DH. Posicionamento da pelve e lordose lombar em mulheres com incontinência urinária de esforço. Fisioter. Pesqui. 2010; 17( 2):130-35. 


\section{Sandra Beatriz Aires dos Santos}

Endereço para correspondência - Rua Aristides Lobo, 148. Bairro Passo da Areia, CEP 97020-080, Santa Maria, RS, Brasil.

E-mail: sandra_aires1@hotmail.com

Lattes: http://lattes.cnpq.br/9999305415699688

Hedioneia Maria Foletto Pivetta - hedioneia@yahoo.com.br

Ana Fátima Viero Badaró - badaroana@uol.com.br

Enviado em 26 de dezembro de 2013.

Aceito em 30 de junho de 2014. 